\title{
International Journal of Food, Nutrition and Public Health
}

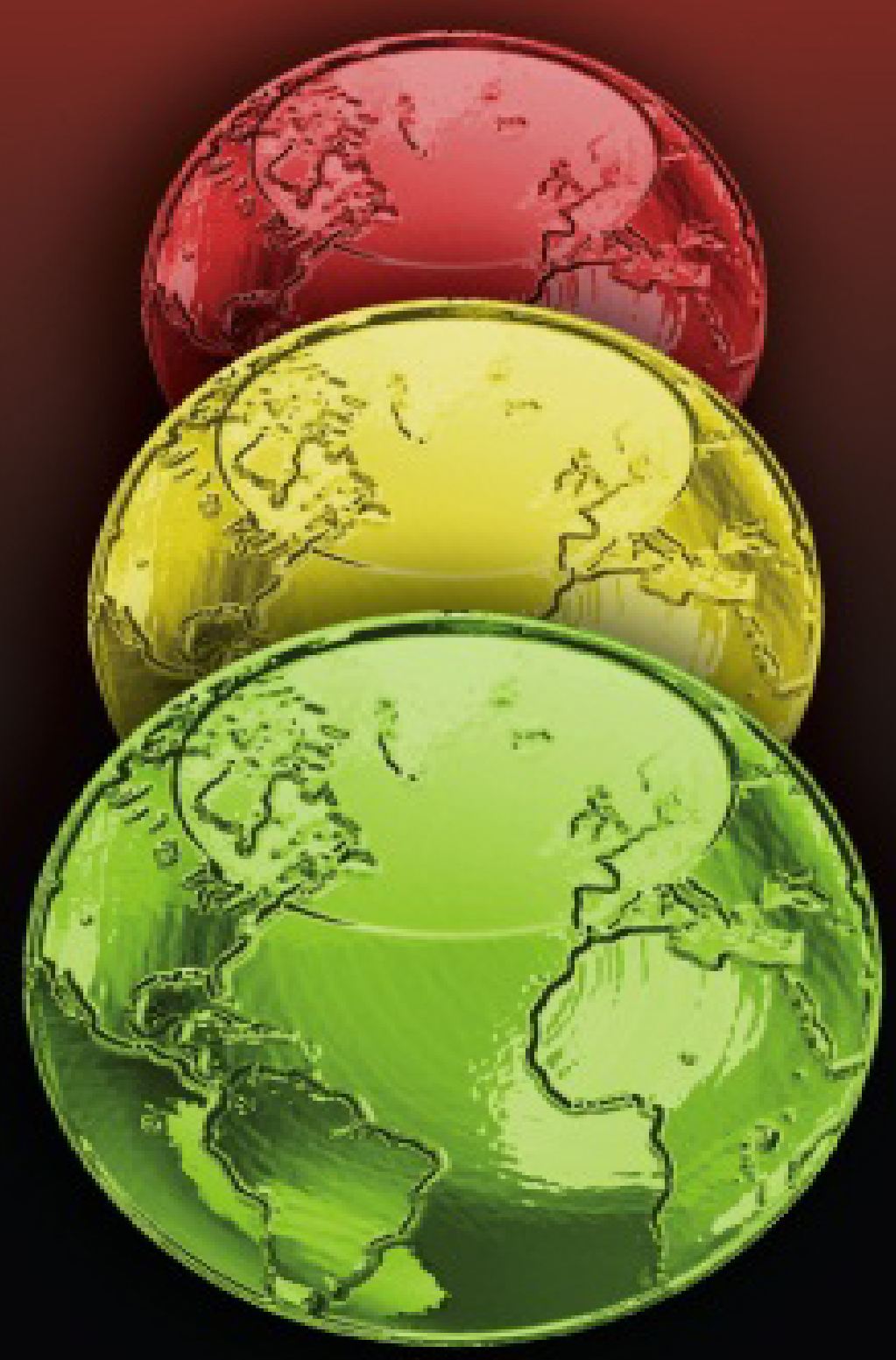

(wanse) www.wasd.org.uk 


\section{CLEAN EATING AN THE RISK OF DISI}

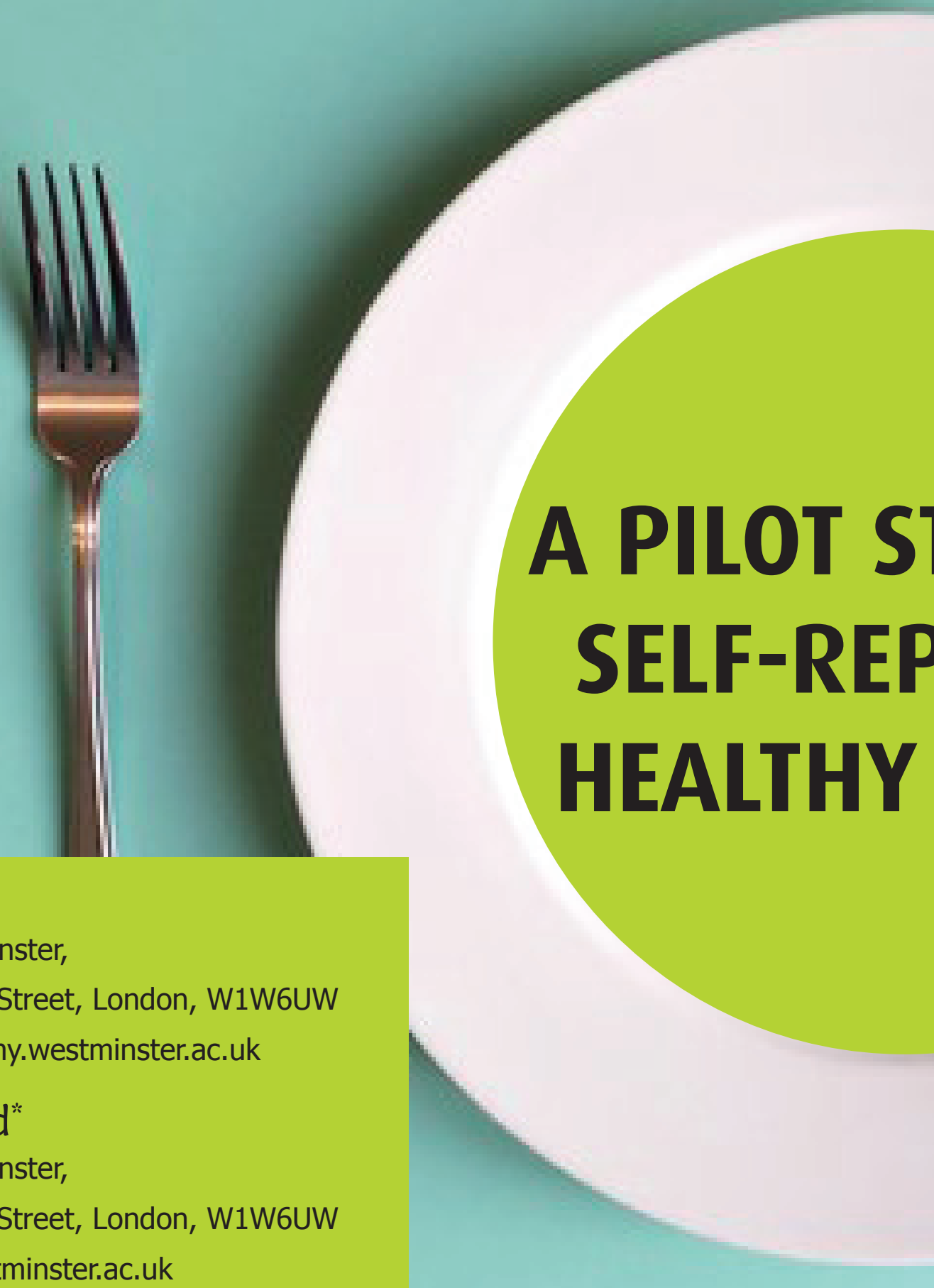

Emily Fivian University of Westminster, 115 New Cavendish Street, London, W1W6UW Email:w1496368@my.westminster.ac.uk

Carly J. Wood" University of Westminster, 115 New Cavendish Street, London, W1W6UW Email: c.wood@westminster.ac.uk 


\section{THE ROLES OF SOCIAL MEDIA}

\section{SELF-ESTEEM IN ORDERED EATING}

\section{UDY OF \\ ORTED EATERS}

\section{BACKGROUND}

Clean eating is a dietary trend focused on the avoidance of unhealthy foods. Social media encourages these highly restrictive diets and this can lead to eating disorders and low self-esteem. This study examines the influence of dietary classification, social media use, diet quality and self-esteem on eating disorder risk amongst a group of self-reported healthy eaters.

\section{METHODS}

A total of 41 participants completed an online survey examining diet quality via the healthy eating index, and eating disorder risk using the eating disorder examination questionnaire (EDE-Q). Participants were also asked to complete Rosenberg's self-esteem scale and reveal whether they defined their diet as either 'clean' or 'pure'; this is reflective of the trend of clean eating. Participants also categorised the time spent on social media each day.

\section{RESULTS}

Independent t-tests revealed that participants who categorised their diet as 'clean' had significantly higher self-esteem (SE) $(\mathrm{t}(39)=2.729$; $p=.009)$; while greater time on social media was associated with elevated eating disorder risk (t(39) $=-2.99 ; p=.005)$ and poorer SE $(\mathrm{t}(39)=-3.01 ; p=.005)$. Multiple linear regression revealed that social media usage was a significant predictor of eating concern $(B=.419$; $p=.01$ ), while SE significantly predicted eating restraint $(B=-.423 ; p$ $=.03)$, shape concern $(B=.217 ; p$ $=.04)$, weight concern $(B=-.454$; $p=.008)$, and the global EDE-Q score $(B=-.437 ; p<.01)$.

\footnotetext{
${ }^{\text {*COrresponding Author }}$
} 


\section{CONCLUSIONS}

Both social media usage and self-esteem might play a key role in the development of eating disorders in a group of self-reported healthy eaters, with high social media usage also influencing self-esteem. Future research should examine how social media could be used to promote good self-esteem and thus reduce eating disorder risk.

\section{KEYWORDS}

Eating disorder; social media; self-esteem; clean eating; dietary trends; eating concern

\section{BIOGRAPHY}

Emily Fivian has a 1st class BSc in Human Nutrition from the University of Westminster, and will shortly be undertaking a Masters' Degree in Public Health at Imperial College. Her research interests are focused on the complexities behind health advice in modern day society and the associated eating behaviours and eating disorder risks. Following completion of her Masters' degree, Emily wishes to pursue an academic career in public health research in order to uncover global health innovations that have the potential to improve health outcomes. ating regulation is a key health priority in modern society, with many policies aimed at improving nutritional health (Lake et al., 2007). Over the past decade the western-world has witnessed an increasing prevalence of eating disorders (EDs). In the UK, over I.25 million people are directly affected, a figure that is likely to be an underestimation due to the large number of people who do not seek help (Anorexia and Bulimia Care, 2015; Smink et al., 2012). EDs are severe psychiatric illnesses associated with numerous negative outcomes. The DSM-5 recognises three primary EDs: anorexia nervosa (AN), bulimia nervosa, and binge-eating disorder (NIMH, 20I6). 'Orthorexia Nervosa' is not currently formally recognised as an ED but it is characterised by an obsession over extremely healthy or biologically 'pure' food, which leads to dietary restriction (Zamora et al., 2005). There are multiple biopsychosocial influences on the aetiology of EDs including the sociocultural idealisation of thinness variables through media exposure, personality traits, such as self-esteem and perfectionism, genetics and dieting (Culbert et al., 20I5).

To be effective, public policy must consider multiple factors to help the public achieve a good nutritional status; these include socio-economic status, food availability, cultural beliefs and socio-norms. The 'many meanings of food', which include psychological perspectives such as emotional factors, dieting and the restraint theory (Ogden, 2008), further complicate food choice and challenge the effectiveness of public health policies. Defining 'healthy eating' is complex, with revolutions in nutritional science and the increase in available resources for health advice resulting in many mixed messages and a lack of clear and shared standards (De Ridder et al., 2013; Paquette, 2005).

Self-regulation of healthy eating is often accounted for by the categorising of food as 'good' and 'bad', or 'healthy' and 'unhealthy' (Freeland-Graves and Nitzke, 2002; Niva, 2007). This has been recognised by the American Dietetic Association who suggests that categorising foods can foster unhealthy eating behaviours; the association therefore strives to promote health regarding the total diet instead of just particular foods.

In recent years the dietary trend of clean eating (CE) has emerged, referring to the following of a strict diet that embodies an eating behaviour centred around proper nutrition and avoidance of foods that would be deemed unhealthy or impure (Nevin and Vartanian, 2017). Media coverage has referred to the CE trend as part of 'the healthy eating movement' in the UK. Although there is little academic literature on the subject, social-media platforms, such as Instagram, encourage diets that often eliminate food groups and are highly restrictive. The diets are based on little or no scientific evidence and are advocated by those who often do not possess any official training in nutritional science (Turner and Lefevre, 2017). Such diets have also been criticised by health care professionals for their lack of scientific-basis (Dunn and Bratman, 2016).Although long term empirical studies on the topic are limited, anecdotal evidence indicates that this pathological fixation on healthy food can lead to similar medical complications as those found in EDs (Koven and Wabry, 20I5), with negative effects on body image, body satisfaction and self-esteem (SE) (Tiggemann 


\section{BIOGRAPHY CONT.}

and Zaccardo, 2018). This highlights how pathological healthful eating could lead to malnutrition.

The primary aim of this study was to determine how dietary classification and social media usage can influence the diet quality, ED risk and SE of self-reported healthy eaters. The secondary aim was to determine which factors might play the biggest role in the development of an ED amongst a healthy group.

\section{METHODS}

\section{Participants}

Participants were recruited via emails inviting them to take part in the study, advertisements around the University of Westminster campus and on student blackboard sites. The inclusion criteria were that participants must consider themselves as a healthy-eater; participants were excluded if they were currently suffering, or had previously suffered, from an ED. The total sample consisted of $4 \mid$ participants (including 10 males and 31 females). Institutional ethical approval was granted prior to the commencement of the study and all participants provided written informed consent prior to completing the questionnaire.

\section{Online Questionnaire}

After providing consent to take part in the study, participants were emailed a link to access a questionnaire that was hosted by google forms. The questionnaire was divided into four sections, assessing demographics and lifestyle, diet quality, SE and ED risk.

The demographic and lifestyle section of the questionnaire asked participants to provide details of their age, gender and ethnicity. Social media usage was determined by 'time on social media per day' with options of less than 15 minutes, 15 minutes-I hour, I-2 hours and 3 hours or above. Participants were also asked whether they defined their day-to-day diet as 'clean' or 'pure' or 'neither'. 'Clean' and 'pure' were used to represent the trend of CE and condensed into one category; this represented the individual's dietary classification.

Participants' diet quality was assessed via The Healthy Eating Index (HEl-20l0). The HEI-20IO has 12 components, 9 based on diet adequacy and 3 based on moderation. Adequacy questions included total fruit, whole fruit, total vegetables, greens and beans, whole grains, dairy (or dairy alternative, i.e. calcium fortified foods), total protein foods, seafood and plant proteins, and the ratio of saturated fatty acid to fatty acids. The moderation questions were based on consumption of refined grains, sodium and empty calories. The index has been determined to quantitatively represent the variety of attributes that make up the 2010 Dietary Guidelines for Americans (Guenther et al., 20I4), which shares common standards with those of UK dietary guidelines. Small adjustments were made to the units of measures to make them universally recognisable. The index provided a score out of 100 , with 100 indicating maximum amount of compliance with the dietary guidelines.
Dr Carly Jane Wood is a lecturer

in Exercise Nutrition and Health at the University of Westminster, and the Food, Nutrition and Public Health Research Group lead. Her research interests are focused on the roles of exercise and nutrition in influencing health outcomes, and how different environments can be used to maximise the health gains derived from taking part in physical activity. Her current research is primarily focused on the role of Green Exercise (physical activity in nature) in modifying responses to stress. 
SE was measured using the Rosenberg Self-Esteem Scale (RSES) (Rosenberg, 1965). The scale assesses global SE and has been shown to be a reliable and valid measure. The RSES is a 10 item scale with items administered using a Likert-type response format from strongly agree to strongly disagree. Five items are reverse scored with overall scores ranging from $0-30$ and higher scores representing better SE.

The Eating Disorder Examination Questionnaire (EDE-Q) is a 30 -item, self-reporting measure that assesses ED psychopathology. The EDE-Q is derived from the well-researched Eating Disorder Examination interview, which is considered the 'gold standard' for assessing the specific psychopathology of EDs (Luce et al., 2008). Research has suggested that the EDE-Q is an acceptable alternative due to its cost-effectiveness and clinical utility (Fairburn and Beglin, 1994). The EDE-Q is scored using a 7-point, forced-choice rating scheme (0-6). Twenty-two questions address the key attitudes and behavioural features of EDs via the use of four sub-scales relating to shape concern, weight concern, eating concern and dietary restraint. These questions all focus on the past 28 days (e.g., on how many of the past 28 days have you had a definite fear that you might gain weight?). The questionnaire generates frequency data providing a global score and individual sub-scale scores. Greater scores indicate a greater amount of problematic eating behaviours. The remaining six questions address ED characteristics in terms of frequency of episodes during the past 28 days. As these questions do not contribute to any of the sub-scale scores or the global score, they were not included in the analysis for this study.

\section{Statistical Analysis}

Independent t-tests were used to compare diet quality, EDE-Q global and sub-scale scores, and SE scores between those who defined their diet as 'clean' or 'pure' and those who did not. Independent t-tests were also used to compare these variables between individuals who reported spending more than and less than one hour per day on social media. This reclassification of responses took place to allow robust statistical analysis to be conducted.

Linear multiple regression was used to assess the impact of SE, social media time, diet quality and diet category on the global EDE-Q score and each of the questionnaire sub-scale scores. The contribution of each model was explored followed by the unique contribution of each of the variables to the overall model. All analyses were conducted in IBM SPSS Statistic 24, with a $p$ value of less than 0.05 indicating statistical significance.

\section{RESULTS}

\section{Participants}

TABLE I shows a summary of the participants' demographic characteristics. Most participants were White British and aged between 18-24 years, spending between 15 minutes to I hour per day on social media. The average diet quality score for the group was $51.63 \pm 18.00$, while the average SE score was $19.37 \pm 18.00$.

\section{Clean Eating}

Independent t-tests revealed no significant differences in diet quality between those who defined their diet as 'clean' or 'pure' and those who did not $(p>.05)$. There was also no significant difference between frequency and severity of disordered eating (DE) behaviours in terms of either the global EDE-Q score, or any of the EDE-Q sub-scales $(p>.05)$ (TABLE 2). For SE, an independent t-test revealed significantly higher scores in those whose defined their diet as 'clean' or 'pure' compared to those who did not (t (39) $=2.729 ; p=.009)$. 
Pilot Study of Self-Reported Healthy Eaters

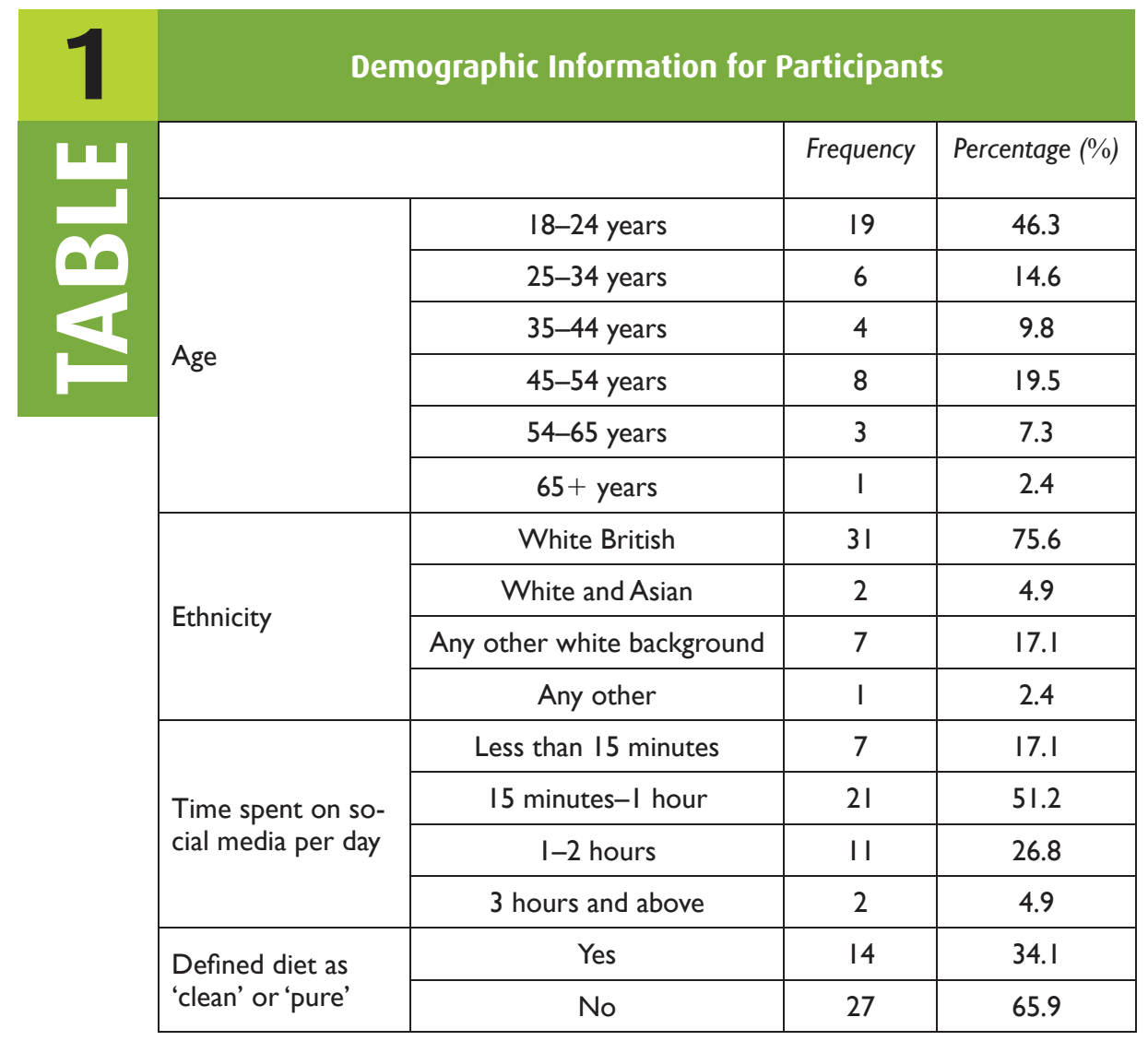

Source: Devised by authors

\begin{tabular}{l}
\hline Influence of Dietary Category and Social Media Time on Diet Quality, Risk of \\
Disordered Eating and Self-esteem
\end{tabular}

*indicates a significant difference between diet category $(p<0.01)$; ${ }^{\#}$ indicates a significant difference due to time spent on social media $(p<0.05)$

Source: Devised by authors 


\section{Social Media}

Independent t-tests revealed no significant differences in diet quality according to time spent on social media $(p>.05)$. There was a significant effect of social media time on the global EDE-Q scores $(t(39)=-2.99 ; p=.005)$. There was also a significant effect of social media on the eating concern $(\mathrm{t}(39)=-2.83 ; p=.0 \mathrm{I})$, shape concern $(\mathrm{t}(39)=-2.55 ; p=.01)$, and weight concern subscales $(\mathrm{t}(39)=-3.01 ; p=.005)$, but not for the eating restraint subscale $(p>.05)$. For both the subscales and global score, more time on social media per day was linked with a greater severity of disordered eating (DE) behaviours (TABLE 2). A significant effect of social media time was also found for $\mathrm{SE}(\mathrm{t}(39)=3.01 ; p=.005)$, with higher SE scores for those who spent less time on social media.

\section{Eating Disorder Risk}

A standard multiple linear regression model was used to predict global EDE-Q scores based on diet quality, SE, dietary classification and social media usage. A significant regression model was found $(f(4,36)=5.060 ; p=.002)$, with an $R^{2}$ of 0.360 , indicating that $36.0 \%$ of the variance in global EDE-Q scores was explained by the model. SE made the strongest unique contribution to the model $(B=-.437, p=.01$ ), with $12.6 \%$ of the variance in global EDE-Q scores being explained by SE. The remaining variables did not make a significant contribution to the global EDE-Q scores (all $p>$.05).

Each of the sub-scale scores were analysed within the same model, with all models except eating restraint being significant: eating restraint: $f(4,36)=$ 2.543; $p=.06 ; R^{2}=.220$; eating concern: $f(4,36)$ $=4.482 ; p=.005 ; R^{2}=.332$; shape concern: $f(4,36)=3.77 \mathrm{I} ; p=.0 \mathrm{I} ; R^{2}=.295$; weight concern: $f(4,36)=5.483 ; p=.00 I ; R^{2}=.379$. SE continued to have a significant unique contribution to the 'eating restraint' $(p=.03)$,'shape concern' $(p=.04)$, and 'weight concern' $(p=.008)$ sub-scales, while time spent on social media made a unique significant contribution to 'eating concern' $(p=.0 \mathrm{I})$ (TABLE 3).

3 Multiple Linear Regression Variables Predicting EDE-Q Sub-scales: Eating
Restraint, Eating Concern, Shape Concern and Weight Concern

*indicates a significant unique contribution to sub-scale score $(p<0.05)$.

Source: Devised by authors 


\section{DISCUSSION}

The primary aim of this study was to determine how dietary classification and social media usage can influence the diet quality, ED risk and SE of self-reported healthy eaters. The findings first revealed that there were no significant differences in the diet quality, global EDE-Q or sub-scale scores between those who defined their diet as 'clean' or 'pure' and those who did not. In fact, the trend of results indicates more favourable scores in the 'clean eating' group for these variables. With the rise in the awareness of the ED of orthorexia nervosa, $C E$ is a new diet term that has been suggested to present a risk to 'healthy' dieters, and is even 'an eating disorder in disguise'. Although there is little academic literature on the topic, with most of the criticism appearing in newspapers and other forms of media from renowned health care professionals, the consensus from the health care authorities, including the ADA, suggests against the use of food categorising and retains an 'all food fits' mantra (Freeland-Graves and Nitzke, 2002). Although it is unclear as to whether CE is a diet or just a term used to express extreme healthiness, adverse social ramifications for $\mathrm{CE}$ have been identified ( $\mathrm{Nev}$ in andVartanian, 2017). However, this study contradicts these suggestions, indicating that this dietary trend does not have adverse dietary implications.

In addition, the CE group also had significantly increased SE. Current literature suggests that dietary restraint can provide strong feelings of self-effica$c y$, a sense of control and achievement that can lead to heightened SE (AbuSabha and Achterberg, 1997). In the face of the obesogenic environment, a heightened degree of control may be integral to allowing some individuals to manage a healthy body weight, and the CE trend could be a suitable method of doing so. However, if an individual's SE is reliant on adhering to dietary rules, problems may arise when dietary rules are broken. In this situation, individuals may be subjected to feeling 'dirty', and the impact this has on their SE might outweigh the enhanced SE felt on a day-to-day basis. On the other hand, if disinhibition of dietary rules never occurred, this could present the social ramification that was discussed by Nevin and Vartanian (2017) and pose risks for obsessive traits similar to those characterised by EDs. Furthermore, the negative associations of CE are the implication that everyone else is 'unclean' and being careless with their bodies and lives (McCartney, 2016). The use of the world 'clean' is therefore unlikely to hold a formal position in health promotion and will remain at its height on social media where it is subject to manipulation and misuse. While this study indicates that $C E$ does not pose an increased risk for $D E$, and may possess some benefits to help people manage their healthy eating and SE, there are many negative notions towards the use of the term and care must be taken as to how and where it is employed.

The study also demonstrated significant differences in both SE and ED risk according to time spent on social media. Those individuals who spent more than one hour per day on social media had a significantly lower SE and higher scores for eating concern, shape concern, weight concern and the global EDE-Q score; this indicates a greater risk of ED development. Social media has previously been found to be a primary predictor for the drive for thinness (Fernandez and Pritchard, 20I2). Increased social media usage and exposure to images of the 'ideal body' can have a negative impact resulting in decreased SE and poor eating behaviours. Body dissatisfaction is not merely a feature of an ED, it is regarded a precursor; therefore the negative influence that social media has needs to be targeted. Social media has a great influential capacity and could be a powerful tool to help increase SE. However, regulation of the portrayal of the ideal body image and usage monitoring need to be conducted to enable this influence to be used positively, particularly in high risk groups such as adolescents.

The secondary aim of this study was to determine which factors might play the biggest role in the de- 
velopment of an ED amongst a group of self-reported healthy eaters. In agreement with the literature (Kelly et al., 2014; Mora et al., 2017), the study presented SE as a significant predictor of EDs, making a significant and unique contribution to global EDE-Q scores and all sub-scales, except for 'eating restraint'. While the direction of cause and effect between the association of low SE, body dissatisfaction and DE is unknown, consistent findings of these associations suggests low SE should be targeted in both the prevention and treatment of EDs. Understanding how SE mediates the development of $D E$ is complex; however, it has been suggested that body image plays a role in this relationship (Brechan and Kvalem, 2015; Kalm and Semba, 2005). This may include the internalisation and investment in the thin-ideal, with a greater importance being placed on body image and appearance as a means for developing an overall opinion of oneself (Shea and Pritchard, 2007). Strategies for promoting good SE and body image are therefore integral to the prevention and treatment of DE.

The study also found that social media usage was a significant predictor of the 'eating concern' subscale. Questions within this sub-scale include aspects of secret eating, feeling guilty about eating, concern about being seen eating, and occasions where thinking about food, eating or calories has affected concentration. Together with images of 'ideal bodies', food is central on social media; often these images are framed positively with the majority depicting fruit and vegetables (Holmberg et al., 2016).Whilst this may hold benefits in the light of the obesity epidemic, constant images of food have the potential to go beyond healthy eating inspiration to an obsession that could affect concentration and other aspects of life.The 'Minnesota Experiment', a human starvation study conducted during World War II (Kalm and Semba, 2005), teaches us of some psychological characteristics of semi-starvation that can be applied to modern-day dieting and are commonly seen in patients with AN. These include obsession over images of food and the collecting of cookbooks and recipes. Many social media platforms provide recipes, catering for all different diet types, such as $C E$, vegan and paleo (Vaterlaus et al., 2015) and have the ability to fuel this kind of obsession. While perhaps useful for guiding healthy food choice, food is often presented in an unrealistic way, with perfectly organised fruit platters; the appearance of the food becomes more important than taste and nutrient quality.

The high levels of social comparison that emerge from social media usage (Bessenoff, 2006), when related to food, could also result in a person feeling guilty about eating something that is not up to the standards portrayed on social media. It is considered normal to think about food when hungry or around meal-times; however, thinking about food at a level that disrupts normal life and affects concentration often distinguishes between those who have an ED and those who do not. Social media's fixation on food could enhance this addiction, and its unregulated role in guiding food choice needs to be further examined. As this study did not assess the exposure specific responses of social media, this explanation remains a theory and provides an avenue for future research.

The main limitation of this study is the method of recruitment. Participants were recruited through opportunistic, non-random sampling, with the majority being students, or colleagues and acquaintances of the authors. As such, it is not possible to confirm that this sample is representative of the general, healthy population. Furthermore, the questionnaire used to assess diet quality, the HEl, could be considered a limitation. The questionnaire was devised around the 2010 dietary guidelines for Americans. While this shares common standards with the UK dietary guidelines, and the authors modified the units of measurements to make them relevant to a UK population, it is possible that the participants found this questionnaire difficult to complete and that it therefore does not accurately represent their dietary consumption. Future 
research in this area should assess whether the specific type and content of social media exposure influences $D E$, as this study was focused on temporal usage rather than content of the media to which the individual was exposed. Intervention strategies to examine whether social media could be used to increase SE and the effect of usage monitoring on both SE and DE would also be valuable.

\section{Public Health Significance}

This research has important implications for public health. The findings demonstrate the potential negative influence that social media can have on both SE and ED risk, and suggest a need for public health initiatives to explore how social media could be used in a positive manner. This is likely to be particularly important for groups such as adolescents who have high social media usage and are at increased risk of poor SE and ED development.

\section{CONCLUSIONS}

In summary, the findings of this study indicate that high social media usage results in reduced SE and heightened ED risk, with both social media and SE significantly contributing to ED risk. Social media is one of the strongest influencers in today's society, rewriting the rules of social interaction, information communication and advertisement strategies (Can and Kaya, 2016). While positive implications of health promotion have been identified, social media still poses ser ious risks to SE and DE behaviours. Future research should therefore seek to examine how social media could be used as a positive tool to promote the development of good SE and thus reduce ED risk.

\section{ETHICAL STATEMENTS}

\section{Acknowledgements: None}

Funding: This research received no specific grant from any funding agency in the public, commercial or not-for-profit sectors.
Availability of Data and Materials: Data available upon request.

Authors Contributions: Emily Fivian designed the study, collected and analysed the data and drafted the first version of the manuscript. Carly Wood advised on the study design, assisted with statistical analysis and drafted the final version of the manuscript.

Conflict of Interest: The authors declare that there is no conflict of interest.

Consent for Publication: Both Emily Fivian and Carly Wood have given consent for manuscript submission.

Ethical Approval: Ethical approval was granted by the University of Westminster Ethics Committee (application number: 20I7/8-UG I2).

\section{REFERENCES}

AbuSabha, R. and Achterberg, C. (1997) Review of Self-Efficacy and Locus of Control for Nutrition and Health-Related Behavior, Journal of the American Dietetic Association, Vol. 97, No. I0, pp. I I22-I I32. doi: 10.1016/ S0002-8223(97)00273-3.

Anorexia and Bulimia Care (2015) Statistics. Available at: http://www.anorexiabulimiacare.org.uk/about/statistics (accessed I4 May 2018)

Bessenoff, G.R. (2006) Can the Media Affect Us? Social Comparison, Self-Discrepancy, and the Thin Ideal, Psychology of Women Quarterly, Vol. 30, No. 3, pp.239-25I. doi: | 0. I | | |/j. | 47|-6402.2006.00292.x.

Brechan, I. and Kvalem, I.L. (20I5) Relationship between body dissatisfaction and disordered eating: Mediating role of self-esteem and depression. Eating Behaviors, Vol. I7, pp.49-58. doi: https://doi.org/10.1016/j.eatbeh.2014.12.008.

Can, L. and Kaya, N. (2016) Social Networking Sites Addiction and the Effect of Attitude towards Social Network Advertising. Procedia - Social and Behavioral Sciences, Vol. 235, pp.484-492. doi: 10.1016/]. SBSPRO.2016.11.059.

Culbert, K.M., Racine, S.E. and Klump, K.L. (20I5)

Research Review:What we have learned about the 
causes of eating disorders - A synthesis of sociocultural, psychological, and biological research. Journal of Child Psychology and Psychiatry, Vol. 56, No. I I, Pp. I I4I-I I 64. doi: I0.1 I I I/jcpp. I244 I.

De Ridder, D., DeVet, E., Stok, M., Adriaanse, M. and De Wit, J. (20I3) Obesity, overconsumption and self-regulation failure: the unsung role of eating appropriateness standards. Health Psychology Review, Vol. 7, No. 2, pp. I46-165. doi: I0.1080/I7437| 99.2012 .706987 .

Dunn, T.M. and Bratman, S. (20I6) On orthorexia nervosa:A review of the literature and proposed diagnostic criteria. Eating Behaviours, Vol. 2 I, No. I, pp. I I-I7. doi:0.1016/J.EATBEH.20I5.12.006.

Fairburn, C.G. and Beglin, S.J. (1994) Assessment of eating disorders: Interview or self-report questionnaire?, International Journal of Eating Disorders, Vol. I6, No. 4, pp.363-370. doi: 10.1002/1098-108X (I994I2) I6:4<363::AID-EAT2260 I60405>3.0.CO;2-\#.

Fernandez, S. and Pritchard, M. (20I2) Relationships between self-esteem, media influence and drive for thinness. Eating Behaviors, Vol. 13, No. 4, pp.32I-325. doi: I0.I0I6/J.EATBEH.20I2.05.004.

Freeland-Graves, J. and Nitzke, S. (2002) Position of The American Dietetic Association:Total Diet Approach to Communicating Food And Nutrition Information. Journal of the American Dietetic Association, Vol. I02, No. I, pp. I00-108. doi: I0.1016/ S0002-8223(02)90030-I.

Guenther, P.M., Kirkpatrick, S.I., Reedy, J., Krebs-Smith, S.M., Buckman, D.W., Dodd, K.W., Casavale, K.O. and Carroll, R.J. (20I4) The Healthy Eating Index-20I0 Is a Valid and Reliable Measure of Diet Quality According to the 2010 Dietary Guidelines for Americans', Journal of Nutrition, Vol. I44, No. 3, pp.399-407. doi: 10.3945/ jn. I I3.183079.

Holmberg, C., Chaplin, J.E., Hillman, T. and Berg, C. (2016) Adolescents' presentation of food in social media:An explorative study. Appetite, Vol. 99, Pp. I2 I-129. doi:I0.1016/J.APPET.2016.01.009.

Kalm, L.M. and Semba, R.D. (2005) They starved so that others be better fed: remembering Ancel Keys and the Minnesota experiment, The Journal of Nutrition, Vol. I35, No. 6, pp. I347-I 352. doi.org/10.1093/jn/I35.6.1347.

Kelly,A.C.,Vimalakanthan, K. and Carter, J.C. (20I4) Understanding the roles of self-esteem, selfcompassion, and fear of self-compassion in eating disorder pathology:An examination of female students and eating disorder patients,

Eating Behaviors, Vol. I5, No. 3, pp.388-39I. doi: 10.10 I6/J.EATBEH.2014.04.008.

Koven, N.S. and Wabry, A. (20I5) The clinical basis of orthorexia nervosa: Emerging perspectives. Neuropsychiatric Disease and Treatment, Vol. 18, No. I, pp.385-394. doi:I0.2 I47/NDT.S6I 665.

Lake, A.A., Hyland, R.M., Rugg-Gunn, A.J.,Wood, C.E., Mathers, J.C. and Adamson, A.J. (2007) Healthy eating: Perceptions and practice (the ASH30 study). Appetite, Vol. 48, No. 2, pp. 176-182. doi: 10.1016/j. appet.2006.08.065.

Luce, K.H., Crowther, J.H. and Pole, M. (2008) Eating Disorder Examination Questionnaire (EDE-Q): Norms for undergraduate women. International Journal of Eating Disorders, Vol. 4I, No. 3, pp.273-276. doi: I0.1002/ eat.20504.

McCartney, M. (2016) Clean eating and the cult of healthism. The British Medical Journal, Vol. 354, p.i4095. doi: 10.1 136/bmj.i4095.

Mora, F., Fernandez Rojo, S., Banzo, C. and Quintero, J. (2017) The impact of self-esteem on eating disorders', European Psychiatry, Vol. 4I, p.S558. doi: https://doi. org/10.1016/j.eurpsy.2017.01.802.

Nevin, S.M. and Vartanian, L.R. (20I7) The stigma of clean dieting and orthorexia nervosa. Journal of Eating Disorders, Vol. 5, No. I, p.37. doi: I0.I I86/s40337-0I70168-9.

Niva, M. (2007) All foods affect health: Understandings of functional foods and healthy eating among healthoriented Finns. Appetite, Vol. 48, No. 3, pp.384-393. doi:10.1016/J.APPET.2006.10.006.

Ogden, J. (2008) The Many Meanings of Food and their Impact on Eating Behaviour. In Buckroyd, J. and Rother, S. (Eds): Psychological Responses to Eating Disorders and Obesity: Recent and Innovative Work. London:Wiley.

Paquette, M.C. (2005) Perceptions of Healthy Eating State of Knowledge and Research Gaps. Canadian Journal of Public Health, Vol. 96, No. 3, pp.s I 5-s I9. doi: 10.2307/41994467.

Rosenberg, M. (1965) Society and the Adolescent Self-Image, Princeton: Princeton University Press.

Shea, M.E. and Pritchard, M.E. (2007) Is self-esteem the primary predictor of disordered eating? Personality and Individual Differences, Vol. 42, No. 8, pp. I 527-1537. doi: I0.10 |6/J.PAID.2006.10.026. 
Smink, F.R.E., van Hoeken, D. and Hoek, H.W. (20I2) Epidemiology of Eating Disorders: Incidence, Prevalence and Mortality Rates. Current Psychiatry Reports, Vol. I4, No. 4, pp.406-4I4. doi: I0.1007/s I 1920-012-0282-y.

The National Institute of Mental Health (NIMH) (20I6) Eating Disorders, National Institute for Mental Health; last updated February 2016.Available via: https://www. nimh.nih.gov/health/topics/eating-disorders/index.shtml (accessed I4 May 2018).

Tiggemann, M. and Zaccardo, M. (2018) Strong is the new skinny: A content analysis of \#fitspiration images on Instagram. Journal of Health Psychology, Vol. 23, No. 8, Pp.1003-I0II. doi: I0.1 I77/I359105316639436.
Turner, P.G. and Lefevre, C.E. (20I7) Instagram use is linked to increased symptoms of orthorexia nervosa. Eating and Weight Disorders, Vol. 22, No. 2, pp.277-284. doi: 10.1007/s405 I9-017-0364-2.

Vaterlaus, J.M., Patten, E.V., Roche, C. and Young, J.A. (20I5) \#Gettinghealthy:The perceived influence of social media on young adult health behaviors. Computers in Human Behavior, Vol. 45, pp.I5I-157. doi: I0.1016/J. CHB.2014.12.013.

Zamora, C., Bonaechea, B., Sanchez, G. and Rial, R. (2005) Orthorexia nervosa. A new eating behavior disorder? Actas Espanolas de Psiquiatria, Vol. 33, No. I, pp.66-68. 